\title{
Wireless automatic router in the application of wireless meter reading system
}

\author{
Xingquan $\mathrm{Li}^{1,2, *}$, Jian $\mathrm{Liu}^{3}$, Chang $\mathrm{Guo}^{2}$, Sijing $\mathrm{Gao}^{2}$ \\ ${ }^{1}$ Key Laboratory of Renewable Energy Utilization Technologies in Buildings, Ministry of Education, Jinan, China, \\ ${ }^{2}$ School of Thermal Energy Engineering, Shandong Jianzhu University, Jinan, China \\ ${ }^{3}$ Engneering Training Center,Shandong University,Jinan, China \\ *Corresponding author \\ xingquanli@msn.com, liujian@sdu.edu.cn,guochangcs@126.com,gaosijing@126.com
}

\begin{abstract}
This article describes the Applications of Wireless automatic routing in Wireless meter reading system. Focus is Wireless technology and wireless automatic routing mechanism implementation.
\end{abstract}

Keywords-Wireless automatic routing; Wireless technology

\section{FOREWORDS}

At present, artificial door meter reading is given priority to other meter reading methods in China which has the following disadvantages: door meter reading brings security problem; Statistical data is not accurate, there are man-made errors; Operation is difficult to standardization which causes unknown loss increase; Data acquisition is not seasonable, system status is unable to real-time reflected, and the effective monitoring cannot be carried on with the user behavior; It causes taking artificial, high cost and low efficiency.

In recent years, the development of remote automatic meter reading system, to a certain extent, solves the problem that maintain a meter reading brought about, it takes the scheme for wired mode. Although remote meter reading way is more reliable than the wireless meter reading way, but there are also some problems: the wiring installation is complex, construction period is long, engineering installation and maintenance cost high, System is difficult to expand and upgrade, and it has poor compatibility with other network . Especially for some old building renovation project, rewiring is not realistic. With the development of wireless technology, wireless short distance communication reliability have a lot of security, application in residents wireless meter reading can guarantee the reliability of wireless communication. In this paper, the wireless automatic routing technique is introduced. That is to say, according to the actual application, we need to research and develop a set of practical stronger automatic routing mechanism which has been applied in the wireless gas meter reading system, the running effect is better, and achieve the expected purpose.

\section{SYSTEM STRUCTURE}

Wireless meter reading system taking wireless gas meter reading system as an example is introduced in this paper. This system consists of direct reading wireless remote transmission gas meter, meter reading machine, monitoring center parts. System structure is as follows:

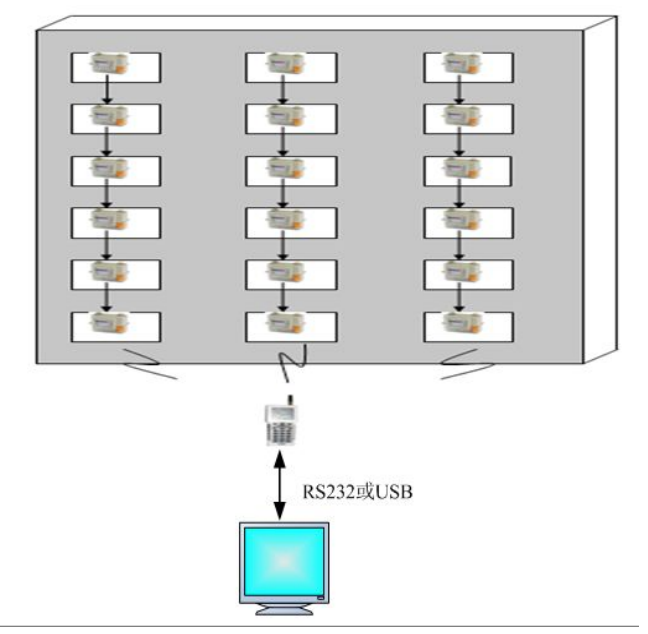

Figure 1 System structure

In this system, each floor of each from the top to the lowest layer composed of a link, such as there are 3 doors each floor in the building, the building has three links, the top household meter is in the lowest level meter of the link.. Meter reading process is as follows:

Meter readers go to the site and directly read the highest level meter of each link with the hand-held meter reading machine, namely the building bottom meter, which can record all the meter data of the link, each layer meter contains all meter data information above the layer, if the top meter has malfunctions, we can get data information of layer 2 and above with the secondary meter.

After the meter read, hand-held meter reading machine connect to computer, the data will be input to the place of business charge management software, then we can carry on statistical analysis and charge.

\section{IMPLEMENTATION BACKGROUND OF THE WIRELESS AUTOMATIC ROUTER}

The wireless data chip of the system adopts ChipCon's product which is the one with a high level of integration, multi-channel RF transceiver. Based on the IEEE802.15.4 standard it is designed for low power wireless applications and has stable operation, high sensitivity, the transmission distance, especially suitable for short-range data 
transmission. In this system, the work frequency point is $470 \mathrm{MHz}$, with modulation mode of FSK mode, transmitting power of $+10 \mathrm{~mW}$ and receiving sensitivity of $-118 \mathrm{dbm}$.

Combined with the practical application, wireless automatic routing mechanism is a set of agreement and Conform to the industry application.

ZigBee technology is a kind of the wireless communication technology providing control or sensor and other electronic components wireless connection. IEEE802.15.4 Group and ZigBee Union established ZigBee standard. ZigBee standard is a developing, low cost, low power consumption, low power, short-range wireless communication standard. The biggest advantage of standard ZigBee is that it has a complete set of AD hoc network protocol composition honeycomb type of AD hoc network system, make point and point correlate to each other, better generality. The disadvantage is that $\mathrm{AD}$ hoc network protocols need tens of $\mathrm{KB}$ of storage space and practicality is not strong in a particular application because of its versatility taking into account up to 65535 nodes.

In practical applications, the more the wireless automatic routing node, the more complex system configuration, then maintenance demand is higher. So wireless automatic routing scheme adopted the advantages of ZigBee agreement, combined with the practical application, and a set of clear concept, simple, practical stronger system was designed in the meter reading system.

IV. IMPLEMENTATION PRINCIPLE OF THE WIRELESS AUTOMATIC ROUTER

- Wireless gas meter is based on the link of automatic routing; each gas meter store a link table, gas meter list is same in the same link. When one gas meter fault need to change, just put the new one's link address set identical to the original;

- Each link table meter from the top to the lowest layer, the top layer for level 1 meter, in turn down to level 2 meter, 3 level meter... , the lowest layer for the highest level meter;

- Level 1 meter make a pass actively every day, the process as follow: 1 level meter hands data to level 2 meter, level 2 meter will pass 1,2 level meters' data to the 3 level table, level 3 meter will hand 1, 2, 3 level meters' data to level 4 meter, and the like, in the end all meter data are handed to the lowest level meter;

- If level 1 meter malfunction cannot pass actively, the level 2 meter passes on automatically;

- Any meter malfunction in link does not affect the entire link operation;

- Bottom meter can save all meter recent 3 times of historical data this link;

- $\quad$ Each link can accommodate most 32 meters;

- Meter reading machine can wake up the bottom gas meter, record all the data of the bottom gas meter by the wireless any time, and allow meter reading machine directly one to one copy each gas meter data;

- Meter reading machine by the wired mode (such as RS232, USB, etc.) record and input the data to the data center in the computer system.

\section{V.SOLVING MECHANISM OF KEY PROBLEM}

\section{A. Signal collision avoidance system}

Each of each floor from the top to the lowest layer make up a link, compose of wireless automatic routing system, in the practical application, wireless signal may influence each other between adjacent building. In order to guarantee the reliability of the system, signal collision avoidance system is indispensable. This application adopts the signal detection mechanism, namely, any one handed the meters in the pass down the time, first detection air have wireless signal, and if there is, it needs to wait for a certain time (in the applications, the waiting time for a complete link pass the longest time), repeat the above steps until no other link recursion pass data to be able to pass preach.

\section{B. Link transmission integrity safe guard mechanism}

In the practical application, there might be a link to a certain watch low battery or malfunction, when the phenomenon appears can't interrupt the link of the signal transmission, so this wants to have a link transmission complete safeguard mechanism. First of all, the mechanism base on link in adjacent 2 watch will not occur at the same time under the premise of fault, in this mechanism, considering the link any a watch may malfunction phenomenon, in the whole link several key node should be considered: level 1 table, intermediate stage table, time senior table, summit table.

\section{Low power consumption mechanism design}

In the whole system the design key point of low power is the use of wireless chip WOR (wireless awaken function). When it is in the receiving state, working current is $12.5 \mathrm{~mA}$, if always let them to be in receiving state, the whole system power consumption will be too big, battery power will soon run out if power supply mode for batteries. In order to solve this contradiction, the system work mode can be set to the WOR work mode, wireless chip need to set two time parameter T0, T1 and receiving duty factor d (Duty Cycle), T0 for the longest work Cycle, dT0 for receiving the longest time, the key of reducing the energy consumption is to reduce the Duty ratio d, when comes into the receiving state the leading code should be directed firstly, if there are leading code, testing the correctness of the address and data. Because the data transmission rate of the system is $19200 \mathrm{bps}$, so sending two bytes of precursor code, the receiving time need $1.2 \mathrm{~ms}$, in order to make sure that you can wake up and be able to complete receives a frame 32 bytes of data, there are certain time margin of the system, in this mode, setting wake up once every 5 seconds, time testing whether there is a connection signal in $10 \mathrm{~ms}$, when 
communicating, sending lead code firstly, to ensure the time of awaking the intelligent terminal CC1100 module is 5 seconds. The system uses the mode that sending two 0xaa for lead code, wireless gas meter receiving two continuous 0xaa and two bytes of synchronous word, judging whether the data is their own data then returns the corresponding state information. When Timer starting time, if in the two cycle within $20 \mathrm{~ms}$ have not received synchronous word and frame initial code, it shall be regarded as invalid data then it re-enter WOR state. So settings the average working current of the whole wireless gas meter is below $30 \mathrm{uA}$, the section use four 5-AA alkaline batteries can work more than one year.

\section{SUMMARY}

With the development of intelligent residential area, automatic centralized meter reading is a kind of development trend, and for the completed residential area of the meter reading system transformation, wireless meter reading system has the advantages that don't need wiring, convenient, flexible and high performance.

The wireless meter reading system based on the wireless automatic routing mechanism which the paper introduces has the features that truly achieve remote meter reading "zero error", saving energy, strong anti-interference capability, high reliability, communication mode diversity, data security good, easy installation and maintenance, and so on, is now relatively perfect in functions of direct reading meter reading system.

\section{REFERENCES}

[1] Liang Jieting, microcontrollers principles and applications[M]. Higher Education Press CHEP, 2002.

[2] Li Wenzhong, and Duan Chaoyu, Wireless Network Technology Introduction and Practice [M], BeJing: Beihang University Press, 2007.

[3] Zheng Quanxiang, Wireless Self-organizing Network Technology Practical Tutorial [M], Tsinghua University Press, 2004.

[4] Chipcon, Products from Texas Instruments for cc1101. 2005.

[5] ZigBee Document 053474r06: ZigBee Specification V1.0[S], ZigBee Alliance, July 2005 